\title{
Special Issue of Translational Stroke: Importance of Sex in the Pathophysiology and Treatment of Acute CNS Repair
}

\author{
Helen M. Bramlett
}

Received: 3 May 2013 /Revised: 6 May 2013 / Accepted: 10 May 2013 /Published online: 25 May 2013

(C) Springer Science+Business Media New York 2013

Over the last several decades, basic research studies as well as clinical programs have emphasized the importance of gender on injury mechanisms leading to the devastating consequences of CNS injury. Also, the importance of gender in targeting these complicated pathomechanisms with various therapeutic interventions has also been emphasized. Indeed, several early investigations reported differences in CNS vulnerability between male and female animals having similar types of insults including cerebral ischemia and neurotrauma. Other studies reported the importance of circulating hormones on affecting the response of the brain to damage, thus emphasizing various endogenous protective mechanisms that may be present in some genders. The present issue includes nine papers that report different aspects of gender-specific differences in the CNS response to damage and how this information can help identify novel therapeutic treatments for these devastating disorders. Each article is written by experts in the field and is well organized, clear, and informative. We feel that this special issue is timely and will be of value to many scientists and clinicians working in the field of CNS injury and repair.

This special issue includes several chapters related to gender-specific differences in response to cerebral ischemia and stroke. Alkayed and colleagues provide a timely review regarding the role of soluble epoxide hydrolase, an enzyme responsible for the degradation of vasoprotective eicosatrienoic acids, and its contributions to the sexual dimorphic nature of stroke [1]. Because endothelial dysfunction and alterations in cerebral blood flow play a major role in stroke outcome, sex differences between processes downstream from estrogen

H. M. Bramlett $(\bowtie)$

University of Miami Miller School of Medicine, Miami, FL, USA

e-mail: HBramlett@miami.edu

H. M. Bramlett

Bruce W. Carter Department of Veterans Affairs Medical Center, Miami, Florida, USA including soluble epoxide hydrogenase appear to be a particularly important factor. In another article, Koellhoffer and McCullough discuss estrogen and inflammation and its interaction with aging [2]. Available clinical literature emphasizes that the risk of stroke in elderly women exceeds that of men, and it is clear that in some situations, estrogen can have proinflammatory effects. This observation is in contrast to the fact that women have a lower incidence of stroke throughout most of their life span which is possibly due to the beneficial effects of steroids including estrogen. This chapter therefore addresses the important concept of what therapeutic options can be utilized to improve recovery once the stroke has occurred.

Furthermore, Raval and colleagues focus on mechanisms by which oral contraceptives and smoking-derived nicotine may inhibit estrogen signaling that could be responsible for the severe ischemic damage seen in females where increased risk and severity of cerebral ischemia are appreciated [3]. This chapter summarizes recent laboratory studies including the report that the severity of ischemic damage in the hippocampus is far greater in females simultaneously exposed to oral contraceptives and cigarette smoking compared to nicotine alone. The authors hypothesize that these factors may increase the severity of post-ischemic damage as a consequence of the lack of estrogen signaling in the brain. In an accompanying article, Herson and colleagues next provide a state-of-the-art review of molecular and cell-based mechanisms underlying sex differences in ischemic brain injury and emphasize the importance of moving toward specialized medicine and effective therapeutic interventions in patients of both sexes [4]. The authors provide summaries of published data on biological sex in clinical stroke and brain injury and provide a summary of the sex differences seen in animal models of ischemic stroke, in vitro models of cell death, as well as sex-specific neuronal cell death mechanisms. The authors emphasize PARPdependent cell death as one important aspect of this sexdependent vulnerability pattern seen in some injury models, emphasizing that intrinsic mechanisms other than nonsteroidal factors may contribute to ischemic outcome. 
This special issue also contains chapters specifically targeting the clinically important area of traumatic brain injury. For example, Covassin provides a timely overview of clinical research on the epidemiology of male and female concussion rates and outcomes including symptoms including cognitive dysfunction post-injury [5]. The authors emphasize that on managing sports-related concussion, several important factors including sex differences, risk factors, the clinical presentation, and management are each extremely important as we develop specialized care for our subjects. In addition to reviewing important new strategies for examining computerized neurocognitive testing when assessing concussed student athletes, the authors also emphasize that general trends indicate that female athletes at both collegiate and high school levels are at greater risk for sustaining a concussion compared to male athletes. Potential reasons for female athletes being at a greater risk for sustaining concussions compared to males include anatomical differences such as weaker neck muscles and a smaller neck girth. This possibility may indicate that the female's head may be more susceptible to higher speeds of acceleration and greater displacement. Also, female soccer players have a larger ball-to-head size ratio than male athletes possibly predisposing them to concussions, as well. Elbin and colleagues review literature on multiple concussions and the potential long-term consequences of these injuries [6]. The use of novel brain imaging and electrophysiological technology for assessing impairments from single or multiple concussions is now providing important data that will allow clinicians to better diagnose concussion and provide important guidance in regards to a potential increased vulnerability to repetitive brain injury. The authors emphasize that concussion history should be examined in combination with other demographic risk factors such as sex that have been shown to influence outcomes from sports-related concussion. Currently, the interactions between history of concussion and sex have not been studied. Finally, Fujii and colleagues provide a review on the important topic of subarachnoid hemorrhage. Subarachnoid hemorrhage is a devastating clinical problem that can lead to severe brain swelling and a high frequency of morbidity and mortality [7]. Thus, as with ischemic stroke, the importance of gender on the pathogenesis including vasospasm and potential therapeutic interventions is an important area of subarachnoid hemorrhage research investigation. Of special relevance to this special issue on gender, subarachnoid hemorrhage has been reported to occur more frequently in females than males.

The importance of gender on recovery after spinal cord injury has also been recently highlighted in the literature. Spinal cord injury is a devastating condition that affects thousands of new individuals each year, the majority of which are males. However, the review by Chan and colleagues emphasizes that females tend to be injured later in life, and recent results from experimental studies have suggested that a gender bias in outcome exists that favors females [8]. This gender-specific difference may again involve the effects of the gonadal sex hormones estrogen and progesterone or testosterone. However, since other studies have been unable to show gender differences on outcome in models of spinal cord injury, there is additional work to be done concerning this important question. This timely review discusses these findings and provides explanations for the complexity of this particular research field.

The final chapter emphasizes that gender has a substantial impact on the influence of various anesthetic agents in the brain [9]. Although half the surgical population is female, there is a trend toward exclusion of females in anesthesia-related research studies thereby providing a huge knowledge gap in the scientific literature. This is an important consideration since different anesthetics may have various effects on the CNS depending on whether a male or female individual is being investigated. This review examines the reported sex-specific differences in the CNS's response to anesthesia and emphasizes a need to perform research experiments on both sexes to further evaluate such differences. The search for new knowledge regarding sexspecific mechanisms in terms of CNS injury and repair represents an important goal for future research investigations. We thank all of the authors and reviewers for their outstanding contributions that have led to this special issue.

\section{References}

1. Davis CM, Fairbanks, SL Alkayed, NJ. Mechanism of the sex difference in endothelial dysfunction after stroke. Trans. Stroke Res. 2013, in press.

2. Koellhoffer EC, McCullough LD. The effects of estrogen in ischemic stroke trans. Stroke Res. 2013, in press.

3. Raval AP, Borges-Garcia R, Diaz F, Sick TJ, Bramlett HM. Oral contraceptives and nicotine synergistically exacerbate cerebral ischemic injury in the female brain. Trans. Stroke Res. 2013, in press.

4. Herson, PS, Palmateer J, Hurn PD. Biological sex and mechanisms of ischemic brain injury Trans. Stroke Res. 2013, in press.

5. Covassin T, Elbin RJ, Crutcher, B; Burkhart, S. The management of sport-related concussion: considerations for male and female athletes. Trans. Stroke Res. 2013, in press.

6. Elbin RJ, Covassin T, Henry L, Whalen DJ, Wedge J, Kontos AP. Sport-related concussion: "how many is too many?" Trans. Stroke Res. 2013, in press.

7. Fujii M, Yan J, Rolland WB, Soejima Y, Caner B, Zhang J. H. Early brain injury, an evolving frontier in subarachnoid hemorrhage research. Trans. Stroke Res. 2013, in press.

8. Chan W-M, Mohammed Y, Lee I, Pearse DD. Effect of gender on recovery after spinal cord injury. Trans Stroke Res. 2013.

9. Mawhinney LJ, Mabourakh D, Lewis MC. Gender-specific differences in the central nervous system's response to anesthesia. Trans. Stroke Res. 2013, in press. 